\title{
Railway Track Crack Detection Vehicle
}

\author{
Saurabh Srivastava ${ }^{1}$, Ravi Prakash Chaurasia ${ }^{2}$ Prashant $^{3}$, Syed Imran Abbas ${ }^{4}$, Prashant Sharma ${ }^{5}$, \\ Nitin Kumar Singh \\ Student, $4^{\text {th }}$ Year, Electrical and Electronics Engineering, IMS Engineering College, Ghaziabad, India ${ }^{1,2,3,4,5,6}$
}

\begin{abstract}
In this paper, we presented a railway track crack detection patrolling vehicle. Indian railway has one of the world's largest railway networks comprising $92,081 \mathrm{~km}(57,216 \mathrm{mi})$ of track over a route of $66,687 \mathrm{~km}(41,437 \mathrm{mi})$ and 7,216 stations.So,manualinspectionand detecting a crack on these railways tracks is very tedious process and consumes lot of time and human resource. This paper proposes a cost effective solution to the problem of railway track crack detection utilizing IR sensor array assembly which tracks the exact location of faulty track, then inform to nearby railway station through short messaging application, so that many lives will be saved. There are many advantages with this system when compared with the traditional detection techniques. It includes less cost, low power consumption and less analysis time.
\end{abstract}

Keywords: ZIGBEE transmitter and receiver, Micro-controller, IR Sensor, Track, LCD.

\section{INTRODUCTION}

India is the fourth largest railway network in the world. Indian rail network is still on the growth trajectory trying to fuel the economic needs of our nation.However, in terms of the reliability and safety parameters, we have not yet reached truly global standards.Cracks in rails have been identified to be the main cause of derailments in the past, yet there have been no cheap automated solutions available for testing purposes. On further analysis of the factors that cause these rail accidents, recent statistics reveal that approximately $60 \%$ of all the rail accidents have derailments as their cause, of which about $90 \%$ are due to cracks on the rails either due to natural causes (like excessive expansion due to heat) or due to antisocial elements.

This track detection vehicle system is basically a electronics device that detects the crack in track and provide the location of crack to nearby stations. This method which utilizes simple components inclusive of a ZigbeeTransmitter and Receiver, Micro-controller, IR Sensors, Photodiode and LED display based crack detector assembly is very useful in railway crack detection. This vehicle will work as a patrolling vehicle on the tracks within regular intervals and transmit update information regarding the tracks conditions. If any fault is detected it will transmit the fault location as well as distance of that particular fault from the starting point by means of Zigbee transmitter to nearby stations. Hence, necessary steps canbe taken as soon as possible by railway authorities to overcome the conditions.This setup is very cost effective as well as highly reliable.

The proposed setup would make the inspection and maintenance of railways tracks easier and help them to monitor efficiently by replacing the human inspection which is currently followed. The design of the vehicle and software related to it are very simple and can be easily adopted by the present system. This idea can be implemented in the long run to facilitate better safety standards and provide effective testing infrastructure for achieving better results inthe future.

\section{BLOCK DIAGRAM}

This is the basic block diagram of our proposed project. It shows all the basic fundamental process of working of this patrolling vehicle.

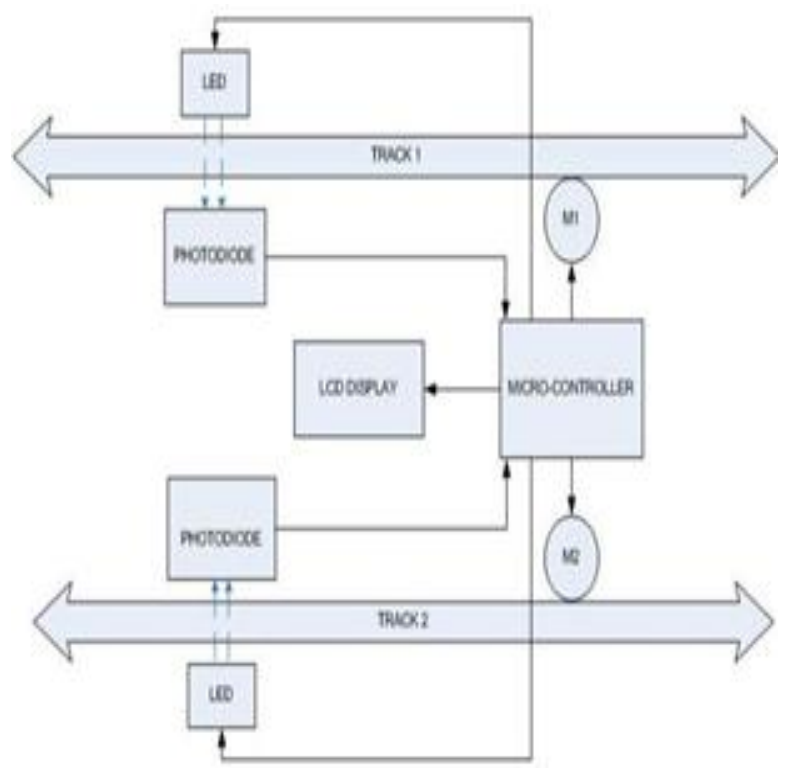

Fig 1: Technical Block Diagram

This assembly consists of two DC motor for movement of vehicle over the railway track. IR (LED) sensors and Photodiode for crack detection. LCD will show the fault location with its distance from starting point. Transmitter will transmit the information to nearby stations. All components are connected to Micro-controller. 


\section{COMPONENTS USED}

1) Micro controller: This section forms the control unitof the whole project. This section basically consists of a Microcontroller with its associated circuitry like Crystal with capacitors, Reset circuitry, Pull up resistors and so on. The Microcontroller forms the heart of the project because it controls the devices being interfaced and communicates with the devices according to the program being.

2) Photodiode: Photodiode is alight sensitive semiconductor diode which converts the light energy into voltage or current based on the mode of operation. In general Photodiodes are operated in reverse bias condition. The clear Photodiode can detect visible and IR rays to limit the Photodiode to detect only IR rays a black cutting is applied to the glass of the Photodiode.

The photodiode allows the current to pass through it if the photodiode is exposed to IR rays and it doesn't allow current to pass through it if no IR rays falls on it. The amount of current passed through the photodiode is directly proportional to amount of IR rays falls on it.

3) Liquid-crystal display (LCD): It is a flat panel display, electronic visual display that uses the light modulation properties of liquid crystals. Liquid crystals do not emit light directly. LCDs are available to display arbitrary images or fixed images which can be displayed or hidden, such as preset words, digits, and 7-segment displays as in a digital clock. They use the same basic technology, except that arbitrary images are made up of a large number of small pixels, while other displays have larger elements.

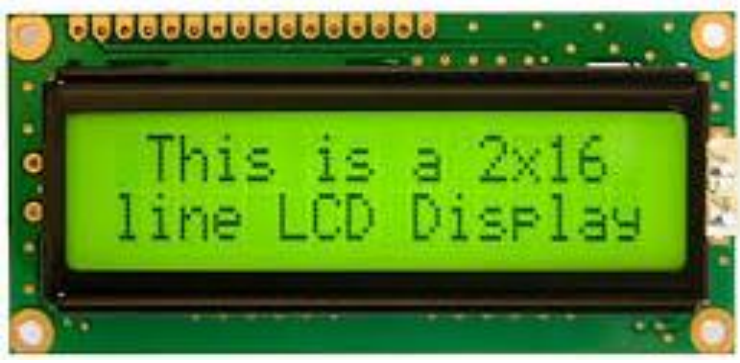

Fig 1: LCD

4) IR Obstacle sensor: This sensor is a short range obstacle detector with no dead zone. It has a reasonably narrow detection area which can be increased using the dual version. Range can also be increased by increasing the power to the IR LEDs or adding more IR LEDs.

The photo below shows my test setup with some IR LED's (dark blue) as a light source and two phototransistors in parallel for the receiver. You could use one of each but I wanted to spread them out to cover a wider area. It has a range of about $10-15 \mathrm{~cm}$ (4-6 inches) with my hand as the object being detected.

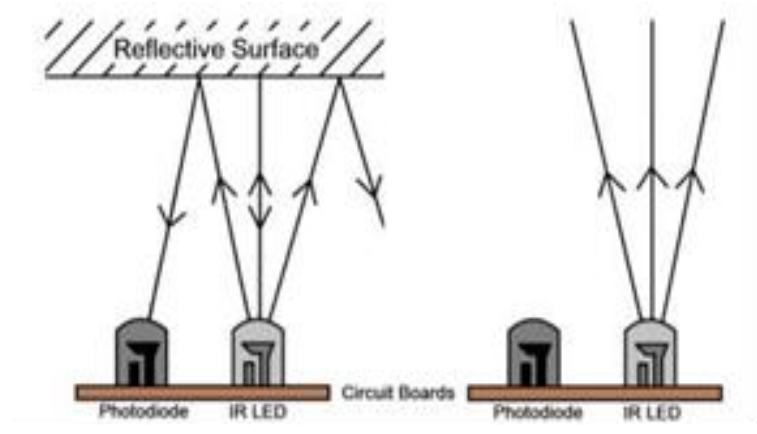

Fig 2: IR obstacle sensor

5) Zigbee Transmitter and Receiver: Zigbee devices are low power, low cost electronic devices which is intended to create personal area networks with low power radios. The major advantage of Zigbee transmitter is that it can transfer data to multiple receivers simultaneously. The physical range of Zigbee transceiver is 10 to 20 metres (approx.). The transmission distance is limited to 10-100 metres line-of-sight due to low power consumption, depending on power output and environmental characteristics. Zigbee devices can transmit data over long distances by passing data through a mesh network of intermediate devices to reach more distant ones.

6) DC Motor: A DC motor's speed can be controlled over a wide range, using either a variable supply voltage or by changing the strength of current in its field windings. It has highest starting torque. So, here to run the vehicle on the tracks we use 2 DC motors.

\section{IV.WORKING}

The vehicle used in this system consists of two IR sensors at its either sides. When vehicle travels over the track, IR transmitter transmits the IR signal continuously. When crack is detected in the track, the transmitted IR signal is passed through crack and received by the receiver. Initially IR receiver is active low, when transmitted IR signal is received by receiver the IR receiver change into active high mode. This signal is feed to the comparator which compares the two signals i.e., transmitted under received and received signal. When the signal is high then comparator pass the signal to the microcontroller which indicates that crack is detected. At this instant motor is stopped. Also the microcontroller receives the distance travelled by the vehicle from a fixed point and it sends this distance of crack to the nearby base station using ZIGBEE Transmitter.

The Zigbee receiver installed at the several base stations receive this information. Then the most nearest base station takes the suitable and necessary steps to prevent any calamities.

\section{FLOW CHART}

The following flow chart showing the step wise working of the railway track crack detection vehicle. 
Vol. 4, Issue 2, February 2017

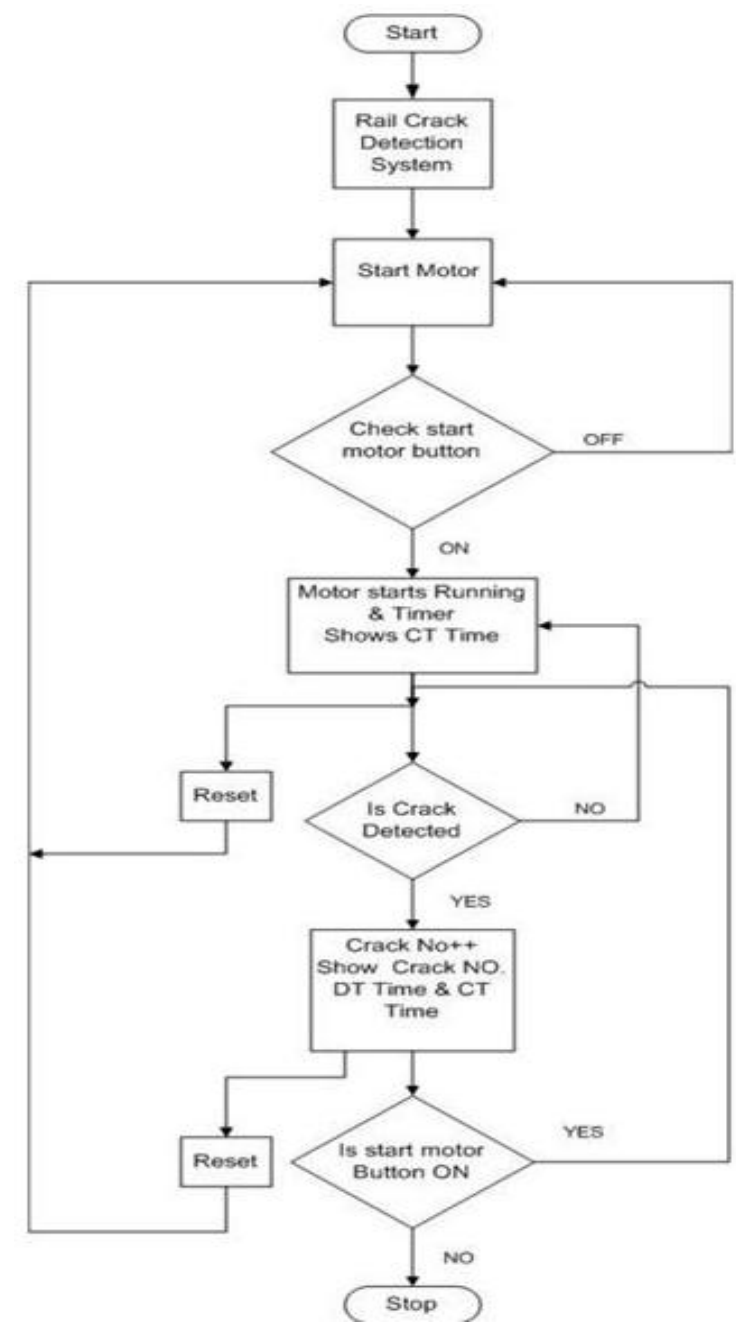

Fig. 3: Flow Chart of Crack detection System

\section{VI.MODEL DESIGN}

The following figure is the actual model of proposed project which shows all the necessary components.

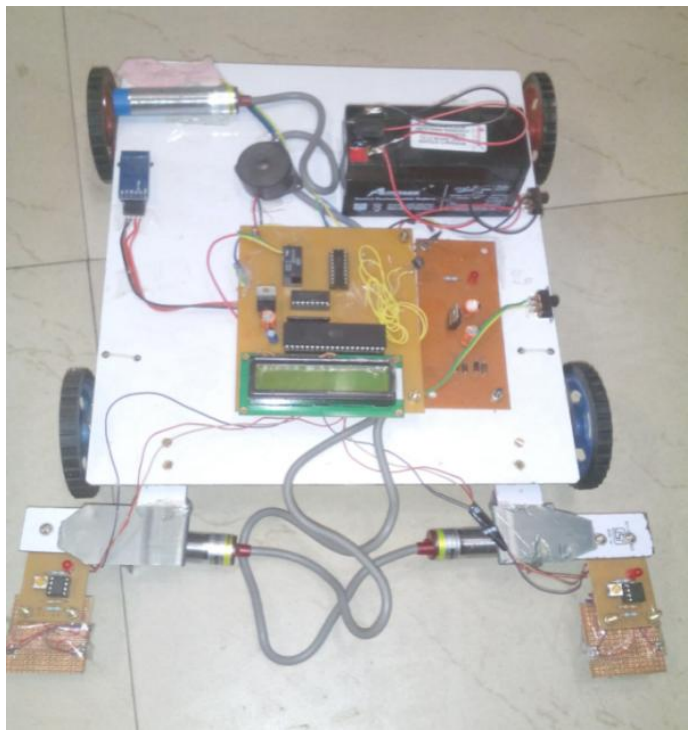

Fig 4: Prototype and components

\section{APPLICATIONS}

Project can be used as patrolling vehicle for inspection of cracks, tear and wear at various places like: Automatic detection of crack on railway tracks.

Calculation of distance of the crack from the origin. Automatic crack detection in forged metal parts. Detection of cracks in concrete pipe.

\section{CONCLUSION}

In this project,by using this Autonomous patrolling vehicle for purpose of railway track inspection and crack detection, it will have a great impact in the maintenance of the tracks which will help in preventing train accidents to a very large extent. The regions where manual inspection is not possible, like in deep coal mines, mountain regions and dense thick forest regions can be easily done using this vehicle.

Cracks in rails have been identified to be the main cause of derailments in the past. Recently on $28^{\text {th }}$ of December,2016 Train no-12987 named Ajmer- Sealdah Express had derailed near Rura, around $70 \mathrm{~km}$ from Kanpur. Fifteen Coaches were derailed in this accident and reported death of 2 people with 48 people was injured.

Hence, owing to the crucial solution of this problem, we have worked on implementing an efficient and cost effective solution suitable for this application. This system automatically detects the faulty rail track without any human intervention. There are many advantages with the proposed system when compared with the traditional detection techniques. The advantages include less cost, low power consumption and less analysis time.Railway track crack detection vehicle is designed in such a way that it detects the cracks or deformities on the track which when rectified in time will reduce train accidents.. By this proposed system, the exact location of the faulty rail track can easily be locatedwhich will mended immediately so that many lives can be saved. By using LED-Photodiode assembly for railway track crack detection system we got accuracy up to $80 \%$.

\section{ACKNOWLEDGMENT}

The authors would like to sincerely thank Dr. (Prof.) Rishi Asthana, HOD, Electrical and Electronics department and our mentor Mr. Gyanesh Singh, Assistant Professor, Electrical and Electronics department, IMS Engineering College for their valuable support and help in this research.

\section{REFERENCES}

[1] Qiao Jian-hua; Li Lin-sheng; Zhang Jing-gang; "Design of Rail Surface Crack-detecting System Based on Linear CCD Sensor", in IEEE Int. Conf. on Networking, Sensing and Control, July 2008, pp. $231-239$

[2] Avinash. V. animireddy and D. ArunaKumari "Automatic Broken Track Detection Using LED-LDR Assembly" in International Journal of Engineering Trends and Technology (IJETT), - July 2013, pp. 289-292. 
[3] Ch. Muneendra Rao , B. R. BalaJaswanth and Ch.Muneendra Rao “ Crack Sensing Scheme in Rail TrackingSystem" in Int. Journal of Engineering Research andApplications, January 2014, pp. 13-18.

[4] R.V.K. Charan "Early detection \& alarming of a transverse crack in a railway track (modified)", International Journal of Scientific \& Engineering Research, January-2013, pp. 234-241.

[5] S. Ramesh, "Detection of Cracks and Railway Collision Avoidance System", International Journal of Electronic andElectrical Engineering, January-2011, pp. 321-327.

[6] SelvamrajuSomalraju, VigneshwarMurali and GouravSaha, "Robust Railway Crack Detection Scheme (RRCDS) Using LEDLDR Assembly", in International conference on recent trends in information technology, March 2012, pp. 477-482

[7] M. Cacciola, G. Megali, D. Pellicanuo, S. Calcagno, M. Versaci, and F. C. Morabito, "Rotating Electromagnetic Field for Crack Detection in Railway Tracks", in PIERS ONLINE, march 2010, pp. 451-459.

[8] S. Ramesh "Detection of Cracks and Railway Collision Avoidance System", International Journal of Electronic and Electrical Engineering ISSN 0974 - 2174 Volume 4, Number 3 (2011)

[9] Paper L. Beales, Track system requirements,_ Railway Group Standards, GC/RT5021, Railway Safety, London, Oct.2003

[10] Journal Richard J. Greene, John R. Yates and Eann A. Patterson, "Crack detection in rail using infrared methods", Opt. Eng. 46, 051013 , May2007.

[11] Komal B. Dandge, Prof. G.R.Gidveer "Automatic Broken Rail Crack Detection Scheme", International Journal of Engineering Research and Applications, ISSN : 2248-9622, Vol. 4, Issue 11(Version - 6), November 2014, pp.01-06.

[12] R.J. Greene, J.R. Yates,E.A. Patterson, "Rail Crack Detection: An Infrared Approach to Inservice Track Monitoring", SEM Annual Conference \& Exposition on Experimental and Applied Mechanics, 2006

[13] Selvamraju Somalraju, Vigneshwar Murali, GouravSaha, Dr. V. Vaidehi, "Robust Railway Crack Detection Scheme (RRCDS) Using LED-LDR Assembly," IEEE Int. Conf. on Networking, Sensing and Control, vol. 6, iss. 3, pg. 453-460 , May2012

[14] Qiao Jian-hua; Li Lin-sheng; Zhang Jing-gang; "Design of Rail Surface Crack- detecting System Based on Linear CCD Sensor," IEEE Int. Conf. on Networking, Sensing and Control, vol. 14, no. 4, pp. 961-970, April 2008. 